\title{
Approximation of nonlinear semigroups and evolution equations
}

\author{
By Jerome A. Goldstein
}

(Received Sept. 23, 1971)

\section{$\S 1$. Introduction.}

Consider a sequence of abstract Cauchy problems

$$
(d / d t) u_{n}(t) \in A_{n}(t) u_{n}(t) \quad(t \geqq 0), u_{n}(0)=x_{n}, n=0,1,2, \cdots
$$

in an arbitrary Banach space $X$. Here $A_{n}(t)$ is for each $t$ a multi-valued function defined on a subset of $X$. We shall show that under suitable hypotheses, if $x_{n}$ converges to $x_{0}$ and if $A_{n}(t)$ converges to $A_{0}(t)$ (in a sense to be made precise below), then $u_{n}(t)$ converges to $u_{0}(t)$.

We first deal with the case when the multi-valued function $A_{n}$ does not depend on $t$ and determines a strongly continuous semigroup of Lipschitzian operators on a subset of $X$. In Section 3, using a generation theorem of Crandall and Liggett [4], we obtain nonlinear generalizations of the TrotterNeveu-Kato approximation theorem for semigroups. These extend results of a number of authors, including Brezis and Pazy [2], Mermin [14], Miyadera [15], and Miyadera and Ôharu [17]. Moreover, our result is best possible in the sense that our sufficient condition is necessary in the linear case.

In Section 4 we establish existence and uniqueness criteria for a special class of time dependent multi-valued Cauchy problems of the form

$$
(d / d t) u(t) \in A(t) u(t) \quad(t \geqq 0), \quad u(0)=x
$$

in a Hilbert space. We also prove an approximation theorem in this situation.

Finally, using existence theorems of Crandall and Liggett [4] and Martin [12], we establish in Section 5 approximation theorems for a class of problems of the form (1.1) in an arbitrary Banach space setting.

\section{§2. Notation.}

Let $X$ be a Banach space with norm $\|\cdot\|$. When $X$ is a Hilbert space, its inner product will be denoted by $\langle\cdot, \cdot\rangle$. "lim" [resp. " $w$-lim"] refers to limit in the norm [resp. weak] topology of $X . \mathscr{Q}(X)$ denotes the set of all subsets of $X . \quad \boldsymbol{R}$ denotes the real numbers, $\boldsymbol{R}^{+}$the nonnegative reals, $\boldsymbol{Z}^{+}$the non- 
negative integers, and $N$ the positive integers. $\operatorname{cl}(S)$ denotes the closure of a set $S$.

For $A, B \subset X \times X, k \in \boldsymbol{R}$ set

$$
\begin{gathered}
A^{-1}=\{(y, x):(x, y) \in A\}, \quad k A=\{(x, k y):(x, y) \in A\}, \\
A+B=\{(x, y+z):(x, y) \in A,(x, z) \in B\}, \\
\mathscr{D}(A)=\{x:(x, y) \in A \text { for some } y\}, \\
\mathscr{R}(A)=\{y:(x, y) \in A \text { for some } x\}, \quad A x=\{y:(x, y) \in A\}, \\
A \mid D=\{(x, y) \in A: x \in D\} \quad \text { where } D \subset X, \\
|D|=\inf \{\|x\|: x \in D\} \text { where } D \subset X .
\end{gathered}
$$

We do not distinguish between (single-valued or multi-valued) functions and their graphs; for instance, we identify the identity operator on $X$ with the diagonal : $I=\{(x, x): x \in X\}$. We do not distinguish between the singleton $\{x\} \in \mathscr{P}(X)$ and the element $x$. For $C_{n} \in X, n \in Z^{+}, \lim _{n \rightarrow \infty} C_{n} \supset C_{0}$ means for each $x_{0} \in C_{0}$, there is an $x_{n} \in C_{n}$ for each $n \in N$ such that $\lim _{n \rightarrow \infty} x_{n}=x_{0}$.

Let $C \in X$. A function $T: C \rightarrow X$ is Lipschitzian if and only if there is a constant $K$ such that $\|T x-T y\| \leqq K\|x-y\|$ for all $x, y \in C$. The smallest possible value of $K$ will be denoted by $\|T\|_{\text {Lip. }}$.

A set $A \subset X \times X$ is called dissipative if and only if for each $\alpha>0,(I-\alpha A)^{-1}$ is a function and $\left\|(I-\alpha A)^{-1}\right\|_{\text {Lip }} \leqq 1$. If $X$ is a real Hilbert space, then $A \subset$ $X \times X$ is dissipative if and only if for all $\left(x_{i}, y_{i}\right) \in A, i=1,2,\left\langle x_{1}-x_{2}, y_{1}-y_{2}\right\rangle$ $\leqq 0$. In other words, $A$ is dissipative if and only if $-A$ is monotone. Also, in the general Banach space case, $A$ is dissipative if and only if $-A$ is accretive, and a characterization of dissipativity can be given in terms of the duality map (or the semi-inner products) of $X$ (cf. Kato [10, p. 141]).

\section{§ 3. Nonlinear semigroups in a Banach space.}

Let $X$ be a Banach space, let $C \subset X$, and let $\omega \in \boldsymbol{R} . \quad T=\left\{T(t): t \in \boldsymbol{R}^{+}\right\}$is a strongly continuous semigroup of type $\omega$ on $C$, or $T \in Q_{\omega}(C)$ for short, if and only if for all $s, t \in \boldsymbol{R}^{+}, x \in C$,

(i) $T(t): C \rightarrow C$,

(ii) $T(t) T(s) x=T(t+s) x, T(0) x=x$,

(iii) $\|T(t)\|_{\mathrm{Lip}} \leqq e^{\omega t}$,

(iv) $\lim _{t \rightarrow 0} T(t) x=x$.

Crandall and Liggett [4] recently established the following definitive generation theorem for semigroups of type $\omega$.

THEOREM 3.1. Let $A \subset X \times X, \omega \in \boldsymbol{R}$ be such that $A-\omega I$ is dissipative and 
$\mathscr{R}(I-\alpha(A-\omega I)) \supset C$ for all sufficiently small $\alpha>0$, where $C=c l(\mathscr{D}(A))$. Then $A$ determines a semigroup $T \in Q_{\omega}(C)$ given by the exponential formula

$$
T(t) x=\lim _{n \rightarrow \infty}\left(I-t n^{-1} A\right)^{-n} x
$$

for all $x \in C, t \in \boldsymbol{R}^{\leftarrow}$.

For our approximation theorems we shall make the following hypothesis.

(*) Let $\boldsymbol{\omega} \in \boldsymbol{R}$. For each $n \in Z^{+}$let $A_{n} \subset X \times X$, let $C_{n}=c l\left(\mathscr{D}\left(A_{n}\right)\right)$, and suppose $A_{n}-\omega I$ is dissipative and $\mathscr{R}\left(I-\alpha\left(A_{n}-\omega I\right)\right) \supset C_{n}$ for $0<\alpha<\alpha_{0}$, where $\alpha_{0}$ is independent of $n$.

In addition to $(*)$ we shall make assumptions about the sense in which $A_{n}$ is to converge to $A_{0}$. Our conclusion will then be:

(**) Let $T_{n}=\left\{T_{n}(t): t \in R^{+}\right\} \in Q_{\omega}\left(C_{n}\right)$ be the semigroup determined by $A_{n}$ as in Theorem 3.1. Then

$$
\lim _{n \rightarrow \infty} T_{n}(t) x_{n}=T_{0}(t) x_{0}
$$

whenever $x_{n} \in C_{n}, \lim _{n \rightarrow \infty} x_{n}=x_{0} \in C_{0} ;$ moreover, the convergence is uniform for $t$ in compact subsets of $\boldsymbol{R}^{+}$.

Here are our main results on semigroups.

THEOREM 3.2. Let (*) hold. Suppose that $\lim _{n \rightarrow \infty} C_{n} \supset C_{0}$; and if $x_{n} \in C_{n}$, $x_{0} \in C_{0}$, and $\lim _{n \rightarrow \infty} x_{n}=x_{0}$, then

$$
\lim _{n \rightarrow \infty}\left(I-\alpha\left(A_{n}-\omega I\right)\right)^{-1} x_{n}=\left(I-\alpha\left(A_{0}-\omega I\right)\right)^{-1} x_{0}
$$

for $0<\alpha<\alpha_{1}, \alpha_{1}$ being independent of $n$. Then (**) holds.

THEOREM 3.3. Let (*) hold. Suppose $C_{n} \supset C_{0}$ for each $n \in N$ and that

$$
\lim _{n \rightarrow \infty}\left(I-\alpha\left(A_{n}-\omega I\right)\right)^{-1} x_{0}=\left(I-\alpha\left(A_{0}-\omega I\right)\right)^{-1} x_{0}
$$

for all $x_{0} \in C_{0}$ and $0<\alpha<\alpha_{1}, \alpha_{1}$ being independent of $n$. Then (**) holds.

Note that

$$
\left(I-\alpha\left(A_{n}-\omega I\right)\right)^{-1}=\beta(I-\alpha \beta A)^{-1}
$$

where $\beta=(1+\alpha \omega)^{-1}$, and so (3.2) holds if and only if

$$
\lim _{n \rightarrow \infty}\left(I-\alpha A_{n}\right)^{-1} x_{n}=\left(I-\alpha A_{0}\right)^{-1} x_{0}
$$

for all $x_{n}, x_{0}$ as in (**) and $0<\alpha<\alpha_{2}$, where $\alpha_{2}$ is independent of $n$. The reason for preferring (3.2) to $\left(3.2^{\prime}\right)$ is to emphasize that the study of the general case (i. e. $\left.T_{n} \in Q_{\omega}\left(C_{n}\right)\right)$ reduces to the contraction case (i. e. $T_{n} \in Q_{0}\left(C_{n}\right)$ ), or, equivalently, it suffices to treat the case that each $A_{n}$ is dissipative.

We shall show that Theorem 3.3 is a special case of Theorem 3.2.

In the linear case (i. e. when each $A_{n}$ and $T_{n}(t)$ are linear and each $C_{n}=X$ ), (3.1) (with $x_{n}=x_{0}$ for all $n$ ) implies (3.3), which in turn implies (3.2), This 
can be seen by noting the formula

$$
\alpha\left(I-\alpha A_{n}\right)^{-1} x=\int_{0}^{\infty} e^{-t / \alpha} T_{n}(t) x d t
$$

for $0<\alpha<1 / \omega$ (or $0<\alpha$ if $\omega \leqq 0$ ) and using the dominated convergence theorem (cf. Chernoff [3], Goldstein [6, p. 39]). It follows that (3.1) is necessary and sufficient for (3.3) (and also for (3.2) in the linear case. In this sense, then, Theorem 3.2 gives the "best" sufficient condition for (**) to hold.

First we prove that Theorem 3.3 is a special case of Theorem 3.2, Let $B_{n}=A_{n}-\omega I$. Whenever $x_{n} \in C_{n}, \lim _{n \rightarrow \infty} x_{n}=x_{0} \in C_{0}$, we have

$$
\begin{gathered}
\left\|\left(I-\alpha B_{n}\right)^{-1} x_{n}-\left(I-\alpha B_{0}\right)^{-1} x_{0}\right\| \leqq\left\|\left(I-\alpha B_{n}\right)^{-1} x_{n}-\left(I-\alpha B_{n}\right)^{-1} x_{0}\right\| \\
+\left\|\left(I-\alpha B_{n}\right)^{-1} x_{0}-\left(I-\alpha B_{0}\right)^{-1} x_{0}\right\|=J_{1}+J_{2},
\end{gathered}
$$

where $J_{1} \leqq\left\|x_{n}-x_{0}\right\|$ and $J_{2} \rightarrow 0$ as $n \rightarrow \infty$ by hypothesis. Hence the hypotheses of Theorem 3.3 imply those of Theorem 3.2, which is what we sought to show.

The point of considering the more complicated condition (3.2) rather than (3.3) is to allow the sets $C_{n}$ to vary as much as possible.

Now we prove Theorem 3.2. The idea of the proof is to deduce the theorem from the Crandall-Liggett generation theorem (Theorem 3.1) by viewing a convergent sequence of semigroups on $X$ as a single semigroup on the space of convergent sequences in $X$. This idea goes back to Kisyński [11].

Let

$$
\mathscr{X}=\left\{\underline{x}=\left\{x_{n}\right\}_{1}^{\infty} \subset X: \lim _{n \rightarrow \infty} x_{n} \text { exists }\right\} .
$$

$\mathscr{X}$ is a (nonreflexive) Banach space with norm $\|\underline{x}\|_{1}=\sup _{n}\left\|x_{n}\right\|$. Define $\mathcal{A} \subset \mathfrak{X}$ $\times \mathscr{X}$ by $:(\underline{x}, \underline{y}) \in \mathcal{A}$ (where $\underline{x}=\left\{x_{n}\right\}_{1}^{\infty}, \underline{y}=\left\{y_{n}\right\}_{1}^{\infty} \in \mathscr{X}$ ) if and only if $x_{n} \in \mathscr{D}\left(A_{n}\right)$, $y_{n} \in A_{n} x_{n}$ for all $n \in N$ and $\lim _{n \rightarrow \infty} x_{n} \in C_{0}$. Let $B_{n}=A_{n}-\omega I, \mathscr{B}=\mathcal{A}-\omega I$. It is easy to see that $(I-\alpha \mathscr{B})^{-1}$ is a function for each $\alpha>0$. To show that $\mathcal{B}$ is dissipative, let $\alpha>0, \underline{x}, \underline{y} \in \mathscr{R}(I-\alpha \mathscr{B})$; then

$$
\begin{gathered}
\left\|(I-\alpha \mathscr{B})^{-1} \underline{x}-(I-\alpha \mathscr{B})^{-1} \underline{y}\right\|=\sup _{n}\left\|\left(I-\alpha \mathscr{B}_{n}\right)^{-1} x_{n}-\left(I-\alpha \mathscr{B}_{n}\right)^{-1} y_{n}\right\| \\
\leqq \sup _{n}\left\|x_{n}-y_{n}\right\|=\|\underline{x}-\underline{y}\|
\end{gathered}
$$

since each $B_{n}$ is dissipative. Hence $\mathcal{B}$ is dissipative on $\mathscr{X}$.

Next we show that $\mathscr{R}(I-\alpha \mathscr{B}) \supset \mathcal{C}$ for all sufficiently small $\alpha>0$, where $\mathcal{C}=\operatorname{cl}(\mathscr{D}(\mathscr{B}))=\operatorname{cl}(\mathscr{D}(\mathcal{A}))$. By hypothesis there is an $\alpha_{2}>0$ such that $\mathscr{R}\left(I-\alpha B_{n}\right)$ $\supset C_{n}$ and (3.2) holds for $n \in Z^{+}$and $0<\alpha<\alpha_{2}$. (Just take $\alpha_{2}=\min \left(\alpha_{0}, \alpha_{1}\right)$.) Let $\underline{x} \in \mathcal{C}$ and $0<\alpha<\alpha_{2}$ be given. For each $n \in N$ choose $y_{n}=\left(I-\alpha B_{n}\right)^{-1} x_{n}$ 
$\in \mathscr{D}\left(A_{n}\right)$. Then $\underline{y}=\left\{y_{n}\right\}_{1}^{\infty}$ is convergent by (3.2), and hence $\underline{y} \in \mathfrak{X}$. Thus: there is a $\underline{y} \in \mathscr{D}(\mathcal{A})$ such that $(I-\alpha \mathscr{B})^{-1} \underline{x}=\underline{y}$. It follows from Theorem 3.1 that $\mathcal{A}$ determines a semigroup $\mathscr{I}=\left\{\mathscr{I}(t): t \in \boldsymbol{R}^{+}\right\} \in Q_{\omega}(\mathcal{C})$. We have $\mathscr{I}(t) \underline{x}=$ $\left\{T_{n}(t) x_{n}\right\}_{1}^{\infty}$ for $t \in \boldsymbol{R}^{+}, \underline{x} \in \mathcal{C}$. Since $\mathcal{I}(t) \underline{x} \in \mathfrak{X}$, it follows that (3.1) holds whenever $\underline{x} \in \mathcal{C}$ and $x_{0}=\lim _{n \rightarrow \infty} x_{n}$.

We next show that if $x_{n} \in C_{n}$ and $\lim _{n \rightarrow \infty} x_{n}=x_{0} \in C_{0}$, then $\underline{x}=\left\{x_{n}\right\}_{1}^{\infty} \in \mathcal{C}$. Let $\varepsilon>0$ be given. For each $n \in Z^{+}$choose $y_{n}=\left(I-t_{n} B_{n}\right)^{-1} x_{n} \in \mathscr{D}\left(A_{n}\right)$; here $\left\{t_{n}\right\}_{0}^{\infty}$ is a sequence of positive numbers which will be chosen presently.

Let $N_{1}=N_{1}(\varepsilon, \underline{x})$ be such that $\left\|x_{n}-x_{0}\right\|<\varepsilon / 3$ for $n>N_{1}$. Chooes and fix $\sigma=\sigma\left(\varepsilon, x_{0}\right)>0$ such that $\left\|\left(I-\sigma B_{0}\right)^{-1} x_{0}-x_{0}\right\|<\varepsilon / 3$; this can be done by [4, Lemma 1.2 (ii)]. Choose $N_{2}=N_{2}(\varepsilon, \underline{x})$ such that $\left\|\left(I-\sigma B_{n}\right)^{-1} x_{n}-\left(I-\sigma B_{0}\right)^{-1} x_{0}\right\|^{\prime \prime}$ $<\varepsilon / 3$ for $n>N_{2}$; this can be done by (3.2). It follows that

$$
\begin{gathered}
\left\|\left(I-\sigma B_{n}\right)^{-1} x_{n}-x_{n}\right\| \leqq\left\|\left(I-\sigma B_{n}\right)^{-1} x_{n}-\left(I-\sigma B_{0}\right)^{-1} x_{0}\right\| \\
+\left\|\left(I-\sigma B_{0}\right)^{-1} x_{0}-x_{0}\right\|+\left\|x_{n}-x_{0}\right\|<\varepsilon
\end{gathered}
$$

for $n>N_{3}=\max \left(N_{1}, N_{2}\right)$. For $1 \leqq n \leqq N_{3}$ choose $t_{n}>0$ so small (by [4, Lemma: 1.2 (ii)]) that $\left\|\left(I-t_{n} B_{n}\right)^{-1} x_{n}-x_{n}\right\|<\varepsilon$, and set $t_{n}=\sigma$ for $n=0$ and for $n>N_{3-}$ It follows that $\underline{y}=\left\{y_{n}\right\}_{1}^{\infty} \in \mathscr{X}$ (since $\lim _{n \rightarrow \infty} y_{n}=\lim _{n \rightarrow \infty}\left(I-\sigma B_{n}\right)^{-1} x_{n}=\left(I-\sigma B_{0}\right)^{-1} x_{0}=y_{0}$ ), $z_{n}=t_{n}^{-1}\left(\left(I-t_{n} B_{n}\right)^{-1} x_{n}-x_{n}\right) \in B_{n} y_{n}$ for all $n \in Z^{+}$, and $\lim _{n \rightarrow \infty} z_{n}=\sigma^{-1}\left(\left(I-\sigma B_{0}\right)^{-1} x_{0}\right.$. $\left.-x_{0}\right) \in B_{0} y_{0}$, so that actually $\underline{y} \in \mathscr{D}(\mathscr{B})=\mathscr{D}(\mathcal{A})$. Furthermore,

$$
\|\underline{y}-\underline{x}\|=\sup _{n}\left\|\left(I-t_{n} B_{n}\right)^{-1} x_{n}-x_{0}\right\| \leqq \varepsilon
$$

by the above estimates. Since $\varepsilon>0$ is arbitrary, it follows that $x \in \mathcal{C}$, and in fact, $\mathcal{C}=\operatorname{cl}(\mathscr{D}(\mathcal{A})$ ).

(We note, parenthetically, that we cannot take $y_{n}=\left(I-\sigma B_{n}\right)^{-1} x_{n}$ for all $n \in Z^{+}$and some $\sigma>0$. To see this let $X=\boldsymbol{R}$ and define $A_{n}=B_{n}$ by $A_{n}(x)$, $=-x / n$ for $|x| \leqq n, A_{n}(x)=-n^{2} x+\left(n^{2}-1\right) x|x|^{-1}$ for $|x|>n$. Then all the hypotheses of Theorems 3.2 and 3.3 hold, and for $x \in \mathscr{X}, \lim _{n \rightarrow \infty}\left(I-\sigma A_{n}\right)^{-1} x_{n}=$ $\left(I-\sigma A_{0}\right)^{-1} x_{0}$ for each $\sigma>0, \lim _{\sigma \rightarrow 0}\left(I-\sigma A_{n}\right)^{-1} x_{n}=x_{n}$ for each $\sigma>0$, and yet. $\inf _{\sigma \geqq 0} \sup _{n}\left\|\left(I-\sigma A_{n}\right)^{-1} x_{n}-x_{n}\right\| \geqq 1$ if $x_{n} \geqq n+1$ for some $n \in N$.)

It only remains to prove the uniformity assertion in $(* *)$. To that end, let $\varepsilon>0, \tau>0, \underline{x} \in \mathcal{C}$ be given. Then by the strong continuity of $\mathscr{I}$,

$$
\|\mathscr{I}(t) \underline{x}-\mathscr{I}(s) \underline{x}\|<\varepsilon / 3
$$

for $0<s, t \leqq \tau$ and $|t-s|<2 \delta=2 \delta(\varepsilon, \underline{x}, \tau)$. Next,

$$
\left\|T_{n}(t) x_{n}-T_{0}(t) x_{0}\right\|<\varepsilon / 3
$$

for $N>N_{t}=N(t, \varepsilon, \underline{x})$ since $\mathscr{T}(t) \underline{x} \in \mathfrak{X}$. Therefore 


$$
\begin{aligned}
& \left\|T_{n}(s) x_{n}-T_{0}(s) x_{0}\right\| \\
& \quad \leqq\left\|T_{n}(s) x_{n}-T_{n}(t) x_{n}\right\|+\left\|T_{n}(t) x_{n}-T_{n}(t) x_{0}\right\|+\left\|T_{n}(t) x_{0}-T_{n}(s) x_{0}\right\|<\varepsilon
\end{aligned}
$$

if $0 \leqq s, t \leqq \tau,|s-t|<2 \delta$, and $n>N_{t}$. Let $k \in N$ be such that $(k-1) \delta<t \leqq k \delta$ and let $N=\max \left\{N_{0}, N_{\delta}, \cdots, N_{k \delta}\right\}$. Then we have

$$
\left\|T_{n}(s) x_{n}-T_{0}(s) x_{0}\right\|<\varepsilon
$$

whenever $0 \leqq s \leqq \tau, n>N$; and $N$ depends only on $\varepsilon, \underline{x}$, and $\tau$. This completes the proof.

REMARK 3.4. Much of the literature on nonlinear semigroups deals with Banach spaces with certain smoothness properties. For instance, many authors only consider spaces with a uniformly convex dual. However, the space $\mathscr{X}$ we constructed in the above proof is always a "bad" space (viz. it is nonreflexive), no matter how "good" the space $X$ is. Thus our proof depends crucially on the availability of a generation theorem which takes place in an arbitrary Banach space.

In the following result we replace condition (3.2) (or (3.3)) by a condition of the form: $\lim _{n \rightarrow \infty} A_{n} y_{0}=A_{0} y_{0}$ for sufficiently many $y_{0} \in \mathscr{D}\left(A_{0}\right)$; the above limit must be carefully interpreted since each $A_{n}$ is in general multi-valued.

THEOREM 3.5. Let (*) hold. Let $D \subset \mathcal{D}\left(A_{0}\right)$ be such that $\operatorname{cl}(D)=C_{0}$, $\mathscr{R}\left(I-\alpha\left(A_{0}-\omega I\right) \mid D\right)$ is dense in $C_{0}$ for all sufficiently small $\alpha>0$, and $D \subset \mathscr{D}\left(A_{n}\right)$ for each $n \in N$. Suppose that for each $y_{0} \in D$, each sufficiently small $\alpha>0$, and each $x_{0} \in\left(I-\alpha\left(A_{0}-\omega I\right)\right) y_{0}$, there is an $x_{n} \in\left(I-\alpha\left(A_{n}-\omega I\right)\right) y_{0}$ such that $\lim x_{n}=x_{0}$. Then (**) holds.

This follows from Theorem 3.3, once we show that (3.3) holds for all sufficiently small $\alpha>0$ and all $x_{0} \in D_{\alpha}$, where each $D_{\alpha}$ is dense in $C_{0}$. Again let $B_{n}=A_{n}-\omega I, n \in Z^{+}$. Let $\alpha>0$ be sufficiently small, and let $D_{\alpha}=$ $\mathcal{R}\left(I-\alpha B_{0} \mid D\right)$. Then $D_{\alpha}$ is dense in $C_{0}$ by hypothesis. Moreover, for each $x_{0} \in D_{\alpha}$, choose $y_{0} \in D$ such that $x_{0} \in\left(I-\alpha B_{0}\right) y_{0}$. Next choose $x_{n} \in\left(I-\alpha B_{n}\right) y_{0}$ such that $\lim _{n \rightarrow \infty} x_{n}=x_{0}$. Then

$$
\begin{gathered}
\left\|\left(I-\alpha B_{n}\right)^{-1} x_{0}-\left(I-\alpha B_{0}\right)^{-1} x_{0}\right\|=\left\|\left(I-\alpha B_{n}\right)^{-1} x_{0}-\left(I-\alpha B_{n}\right)^{-1} x_{n}\right\| \\
\leqq\left\|x_{n}-x_{0}\right\| \rightarrow 0 \quad \text { as } n \rightarrow \infty,
\end{gathered}
$$

and thus (3.3) holds.

REMARK 3.6. The scope of Theorems 3.2 and 3.3 is wider than that of Theorem 3.5. This is true even in the linear case, since there exists a sequence of skew-adjoint (linear) operators $\left\{A_{n}: n \in Z^{+}\right\}$on a Hilbert space $X$ such that $\lim _{n \rightarrow \infty}\left(I-\alpha A_{n}\right)^{-1} x_{0}=\left(I-\alpha A_{0}\right)^{-1} x_{0}$ for all $x_{0} \in X$ and all $\alpha \in \boldsymbol{R} \backslash\{0\}$, but nevertheless $\mathscr{D}\left(A_{0}\right) \cap \bigcup_{n=1}^{\infty} \mathscr{D}\left(A_{n}\right)=\{0\}$ (cf. Goldstein [7]). 
We conclude this section with a result on the convergence of the solutions of the Cauchy problems (1.1) with $A_{n}(t)$ not depending on $t$.

THEOREM 3.7. Let $X$ be reflexive. Let the hypotheses of Theorem 3.2 (or 3.3 or 3.5) hold. Assume additionally that $A_{n} \subset X \times X$ is closed.

(i) If $x_{n} \in \mathscr{D}\left(A_{n}\right), u_{n}(\cdot)$ defined by $u_{n}(t)=T_{n}(t) x_{n}$ is the unique function satisfying: $u_{n}(\cdot)$ is Lipschitz continuous on compact intervals in $\boldsymbol{R}^{+}, u_{n}(\cdot)$ is. strongly differentiable a.e., $u_{n}(0)=x_{n}$, and $(d / d t) u_{n}(t) \in A_{n} u_{n}(t)$ a.e.

(ii) If $x_{n} \in \mathscr{D}\left(A_{n}\right)$ for all $n \in Z^{+}$and $\lim _{n \rightarrow \infty} x_{n}=x_{0}$, then $\lim _{n \rightarrow \infty} u_{n}(t)=u_{0}(t)$ for all $t \in \boldsymbol{R}^{+}$, the convergence being uniform for $t$ in compact subsets of $\boldsymbol{R}^{+}$.

(i) is due to Crandall and Liggett [4, Theorem II], Miyadera [30, Theorem 1]; (ii) follows immediately from Theorem 3.2 (or 3.3 or 3.5). We note, as did Crandall and Liggett [4], that we need not assume that $X$ is reflexive if instead we assume that each $A_{n}$ is single-valued and either linear or continuous. In fact, in the continuous case, we have the following result.

THEOREM 3.8. Let $\left\{A_{n}\right\}_{0}^{\infty}$ be a sequence of continuous operators defined on all of $X$ such that $A_{n}-\omega I$ is dissipative for some real $\omega$ and all $n \in Z^{+}$. The Cauchy problem

$$
(d / d t) u_{n}(t)=A_{n} u_{n}(t), \quad u_{n}(0)=x_{n}
$$

has a unique strongly continuously differentiable solution $u_{n}: \boldsymbol{R}^{+} \rightarrow X$ for each $x_{n} \in X$ and each $n \in Z^{+}$. If

$$
\lim _{n \rightarrow \infty}\left(I-\alpha\left(A_{n}-\omega I\right)\right)^{-1} x_{0}=\left(I-\alpha\left(A_{0}-\omega I\right)\right)^{-1} x_{0}
$$

for all $x_{0} \in X$ and $0<\alpha<\alpha_{0}, \alpha_{0}$ being independent of $x_{0}$, then

$$
\lim _{n \rightarrow \infty} u_{n}(t)=u_{0}(t)
$$

for each $t \in \boldsymbol{R}^{+}$whenever $\lim _{n \rightarrow \infty} x_{n}=x_{0}$; here $u_{n}$ is as above. Moreover, the convergence is uniform for $t$ in compact subset of $\boldsymbol{R}^{\boldsymbol{t}}$.

The proof is the same as that of Theorem 3.2, only instead of using the Crandall-Liggett generation theorem, we use one due to Martin [13] and Webb [22]. We omit the details.

\section{§4. Some time dependent multi-valued equations in Hilbert space.}

Let $H$ be a real Hilbert space. A dissipative subset of $H \times H$ is maximal if it is not properly contained in any other dissipative set.

THEOREM 4.1. Let $\omega \in \boldsymbol{R}$. Let $A \subset H \times H$ be such that $A-\omega I$ is maximal dissipative. Let $F: \boldsymbol{R}^{+} \times \mathscr{D}(A) \rightarrow H$ satisfy a global Lipschitz condition:

$$
\|F(t, x)-F(s, y)\| \leqq K\{\|x-y\|+|t-s|\}
$$


for some constant $K$ and all $(t, x),(s, y) \in \boldsymbol{R}^{+} \times \mathscr{D}(A)$. Then the initial value problem

$$
\begin{gathered}
(d / d t) u(t) \in A u(t)+F(t, u(t)) \quad\left(t \in \boldsymbol{R}^{\dagger}\right), \\
u(0)=x \in \mathscr{D}(A)
\end{gathered}
$$

has a unique solution $u: \boldsymbol{R}^{+} \rightarrow H$ in the following sense:

(i) $u$ is Lipschitz continuous on compact subintervals of $\boldsymbol{R}^{+}, u\left(\boldsymbol{R}^{+}\right) \subset \mathscr{D}(A)$, $u(0)=x$,

(ii) $u$ has a strongly right continuous right derivative satisfying

$$
\left(d^{+} / d t^{\leftarrow}\right) u(t) \in A u(t)-F(t, u(t)) \quad\left(t \in \boldsymbol{R}^{\star}\right),
$$

(iii) the strong derivative $(d / d t) u(t)$ exists and is strongly continuous on $\boldsymbol{R}^{+}$except for at most countably many values of $t$.

The idea behind the proof is the trivial classical trick which converts a nonautonomous ordinary differential equation to an autonomous one (see e.g., $[21$, p. 34]).

Define $F(t, x)=F(-t, x)$ for $t<0, x \in \mathscr{D}(A)$. Let $H_{1}=H \times \boldsymbol{R}$. Vectors in $H_{1}$ will be denoted by column vectors such as $\left(\begin{array}{l}x \\ t\end{array}\right), x \in H, t \in \boldsymbol{R}$. With respect to the inner product

$$
\left\langle\left(\begin{array}{l}
x \\
t
\end{array}\right),\left(\begin{array}{l}
y \\
s
\end{array}\right)\right\rangle_{1}=\langle x, y\rangle+t s,
$$

$H_{1}$ becomes a real Hilbert space. The corresponding norm will be denoted by $\|\cdot\|_{1}$. Define $D \subset H_{1} \times H_{1}$ by

$$
D=\left\{\left(\left(\begin{array}{l}
x \\
t
\end{array}\right),\left(\begin{array}{l}
y \\
1
\end{array}\right)\right): x \in \mathscr{D}(A), t \in \boldsymbol{R}, y \in A x+F(t, x)\right\} .
$$

Then for $\left(\left(\begin{array}{c}x_{i} \\ t_{i}\end{array}\right),\left(\begin{array}{c}y_{i} \\ 1\end{array}\right)\right) \in D, i=1,2$, our hypotheses imply

$$
\begin{aligned}
& \left\langle\left(\begin{array}{c}
x_{1} \\
t_{1}
\end{array}\right)-\left(\begin{array}{c}
x_{2} \\
t_{2}
\end{array}\right),\left(\begin{array}{c}
y_{1} \\
1
\end{array}\right)-\left(\begin{array}{c}
y_{2} \\
1
\end{array}\right)\right\rangle_{1} \\
& \quad=\left\langle x_{1}-x_{2}, z_{1}-z_{2}\right\rangle+\left\langle x_{1}-x_{2}, F\left(t_{1}, x_{1}\right)-F\left(t_{2}, x_{2}\right)\right\rangle \\
& \quad \text { for some } z_{i} \in A x_{i}, i=1,2 \\
& \geqq-\omega\left\|x_{1}-x_{2}\right\|^{2}-2 K\left(\left\|x_{1}-x_{2}\right\|^{2}+\left|t_{1}-t_{2}\right|^{2}\right) \\
& \geqq-\sigma\left\|\left(\begin{array}{c}
x_{1} \\
t_{1}
\end{array}\right)-\left(\begin{array}{c}
x_{2} \\
t_{2}
\end{array}\right)\right\|_{1}^{2}
\end{aligned}
$$

where $\sigma=\omega+2 K$. Hence $B-\sigma I$ is dissipative. Moreover, it is maximal dissipative by a perturbation theorem of Crandall and Pazy [5, p. 403]. It follows from a generation theorem of Crandall and Pazy [5], [18] that $D$ determines a semigroup $S=\left\{S(t): t \in \boldsymbol{R}^{+}\right\} \in Q_{\omega}(c l(\mathscr{D}(A)))$ given by the exponential 
formula of Theorem 3.1. Moreover, it follows easily from the properties that Crandall and Pazy [5], [18] established for $S$ that if we $\operatorname{set}\left(\begin{array}{c}u(t) \\ t\end{array}\right)=S(t)\left(\begin{array}{l}x \\ 0\end{array}\right)$ with $x \in \mathscr{D}(A)$, then $u$ is the unique solution of (4.1), (4.2) in the sense of (i), (ii), and (iii).

The next result is a generalization of Theorem 4.1 to the case when $F$ is multi-valued.

THEOREM 4.2. Let $\omega \in \boldsymbol{R}$. Let $A \subset H \times H$ be such that $A-\omega I$ is maximal dissipative. Let $F: \boldsymbol{R}^{+} \times S \rightarrow \mathscr{P}(H)$ where $\mathscr{D}(A) \subset S \subset H$ satisfy:

(F1) There are constants $M_{1}, M_{2}$, a nondecreasing function $k_{1}$ on $\boldsymbol{R}^{+}$satisfy. ing $k_{1}(r)<1$ for each $r \in \boldsymbol{R}^{+}$, and a function $k_{2}$ on $\boldsymbol{R}^{*} \times \boldsymbol{R}^{+}$to $\boldsymbol{R}^{+}$such that

(i) for each $t_{i} \in \boldsymbol{R}^{+}, x_{i} \in S, y_{i} \in F\left(t_{i}, x_{i}\right), i=1,2,\left\langle x_{1}-x_{2}, y_{1}-y_{2}\right\rangle \geqq-M_{1}\left\{\| x_{1}\right.$ $\left.-x_{2} \|^{2}+\left|t_{1}-t_{2}\right|^{2}\right\}$

(ii) for each $t_{i} \in \boldsymbol{R}^{+}, x_{i} \in \mathscr{D}(A), z_{i} \in A x_{i}, y_{i} \in F\left(t_{i}, x_{i}\right), i=1,2,\left\|y_{1}-y_{2}\right\| \leqq$ $M_{2}\left\{\left|t_{1}-t_{2}\right|+\left\|x_{1}-x_{2}\right\|\right\}+k_{1}\left(\left\|x_{1}\right\|+\left\|x_{2}\right\|\right)\left\|z_{1}-z_{2}\right\|+k_{2}\left(t_{1}, t_{2}\right)$.

(F2) For each $t \in \boldsymbol{R}^{+}$and each $y \in H$, there is a sequence $\left\{n_{i}\right\}$ in $\boldsymbol{R}^{+}$tending to infinity, and there is a sequence $\left\{x_{i}\right\}$ in $S$ such that $y \in F\left(t, x_{i}\right)+n_{i} x_{i}$, $i \in N$. Then the initial value problem (4.1), (4.2) has a unique solution $u$ in the sense of (i), (ii), and (iii) of Theorem 4.1.

The proof is similar to that of Theorem 4.1, but a little more complicated. One shows that $D-\tau I$ is dissipative, where $\tau$ is a suitable constant. To show that $D-\tau I$ is maximal dissipative, one uses a perturbation theorem of Brezis, Crandall and Pazy [1, p. 233]. We omit the details.

REMARK 4.3. Condition (F2) is superfluous if $F$ is single-valued by $[5$, p. 403]. It follows that Theorem 4.1 is a special case of Theorem 4.2.

The next two theorems are approximation theorems in the situation of Theorem 4.1. (We omit the analogous result based on Theorem 4.2.) The basic situation is described as follows:

(+) Let $\omega \in \boldsymbol{R}$. For each $n \in Z^{+}$let $A_{n} \subset H \times H$ be such that $A_{n}-\omega I$ is maximal dissipative. For each $n \in Z^{+}$let $F_{n}: \boldsymbol{R}^{+} \times \mathscr{D}\left(A_{n}\right) \rightarrow H$ satisfy a global Lipschitz condition:

$$
\|F(t, x)-F(s, y)\| \leqq K\{\|x-y\|+|t-s|\}
$$

for some constant $K$ (independent of $n$ ) and all $(t, x),(s, y) \in \boldsymbol{R}^{+} \times \mathscr{D}\left(A_{n}\right)$.

Our conclusion will be :

$(++)$ For $n \in Z^{+}$let $u_{n}$ be the unique solution of the initial value problem

$$
(d / d t) u_{n}(t) \in A_{n} u_{n}(t)+F_{n}\left(t, u_{n}(t)\right) \quad\left(t \in R^{+}\right), u_{n}(0)=x_{n} \in \mathscr{D}\left(A_{n}\right)
$$

in the sense of Theorem 4.1. If $\lim _{n \rightarrow \infty} x_{n}=x_{0}$, then $\lim _{n \rightarrow \infty} u_{n}(t)=u_{0}(t)$ for all $t \in \boldsymbol{R}^{+}$, the convergence being uniform on compact subsets of $\boldsymbol{R}^{+}$. 
Note that if $(+)$ holds, then $u_{n}$ as described in $(++)$ exists by Theorem 4.1.

THEOREM 4.3. Let $(+)$ hold. Suppose that $\lim _{n \rightarrow \infty} C_{n} \supset C_{0}\left(\right.$ where $\left.C_{n}=\operatorname{cl}\left(\mathscr{D}\left(A_{n}\right)\right)\right)$ and suppose that

$$
\lim _{n \rightarrow \infty}\left(I-\alpha\left(A_{n}+F_{n}(t, \cdot)-\sigma I\right)\right)^{-1} x_{0}=\left(I-\alpha\left(A_{0}+F_{0}(t, \cdot)-\sigma I\right)\right)^{-1} x_{0}
$$

for all $x_{0} \in C_{0}$ and all sufficiently small $\alpha>0$; here $\sigma=2 K+\omega$. Then $(++)$ holds.

Let $F_{n}(t, x)=F_{n}(-t, x)$ for $t<0, x \in \mathscr{D}\left(A_{n}\right)$, and let $H_{1}$ be as in the proof of Theorem 4.1. Define $D_{n} \subset H_{1} \times H_{1}$ by $D_{n}=\left\{\left(\left(\begin{array}{l}x \\ t\end{array}\right),\left(\begin{array}{l}y \\ 1\end{array}\right)\right): x \in \mathscr{D}\left(A_{n}\right), t \in \boldsymbol{R}\right.$, $\left.y \in A_{n} x+F_{n}(t, x)\right\}$. Let $E_{n}=D_{n}-\sigma I$. Then $E_{n}$ is maximal dissipative by the proof of Theorem 4.1. It is easy to see that $\left(\left(\begin{array}{l}x \\ t\end{array}\right),\left(\begin{array}{l}y \\ s\end{array}\right)\right) \in\left(I-\alpha E_{n}\right)$ if and only if $s=t-\alpha, y \in x-\alpha\left(A_{n} x+F_{n}(t, x)-\sigma x\right)$; hence $\left(I-\alpha E_{n}\right)^{-1}\left(\begin{array}{l}y \\ s\end{array}\right)=\left(\begin{array}{c}z \\ t\end{array}\right)$ where $t=$ $x+\alpha, z \doteq(1-\alpha \sigma)^{-1}\left(I-\beta\left(A_{n}+F_{n}(t, \cdot)-\sigma I\right)\right)^{-1} y$; here $\beta=\alpha(1-\alpha \sigma)^{-1}$. It follows from Theorem 3.2 that the semigroup determined by $D_{n}$ converges strongly to the semigroup determined by $D_{0}$. Since these semigroups govern (4.3), the conclusion of the theorem follows easily (cf. the last sentence of the proof of Theorem 4.1).

THEOREM 4.4. Let (+) hold. Let $D \subset \mathscr{D}\left(A_{0}\right)$ be such that $\operatorname{cl}(D)=C_{0}$ $\left(=\operatorname{cl}\left(\mathscr{D}\left(A_{0}\right)\right)\right), \mathscr{R}\left(I-\alpha\left(A_{0}-\omega I\right) \mid D\right)$ is dense in $C_{0}$ for all sufficiently small $\alpha>0$, and $D \in \mathscr{D}\left(A_{n}\right)$ for each $n \in N$. Suppose that for each $y_{0} \in D$, each sufficiently small $\alpha>0$, and each $x_{0} \in\left(I-\alpha\left(A_{0}-\omega I\right)\right) y_{0}$, there is an $x_{n} \in\left(I-\alpha\left(A_{n}-\omega I\right)\right) x_{0}$ such that $\lim _{n \rightarrow \infty} x_{n}=x_{0}$. Suppose further that $\lim _{n \rightarrow \infty} F_{n}\left(t, x_{0}\right)=F_{0}\left(t, x_{0}\right)$ for each $t \in \boldsymbol{R}^{\boldsymbol{t}}$ and each $x_{0} \in D$. Then $(++)$ holds.

The proof is essentially the same as that of Theorem 4.3 , but it is based on Theorem 3.5 rather than Theorem 3.2. We omit the details.

\section{§5. Time dependent equations in an arbitrary Banach space.}

The proof of the approximation theorems of Section 3 can be easily adapted to the case of time dependent evolution equations. The existence theorem we shall use is the following result of Crandall and Liggett [4, Appendix to Section 1$]$.

THEOREM 5.1. Let $\omega \in \boldsymbol{R}$. For each $t \in J=[0, T]$ let $A(t) \subset X \times X$ be such that $A(t)-\omega I$ is dissipative. In addition, assume the following conditions.

(i) $\mathscr{D}(A(t))$ is independent of $t$.

(ii) $\mathscr{R}(I-\alpha(A(t)-\omega)) \supset C=\operatorname{cl}(\mathscr{D}(A(0)))$ for $0<\alpha<\alpha_{0}$ and $t \in J$.

(iii) $|A(t) x| \leqq|A(\tau) x|+|t-\tau| L(\|x\|)(1+|A(\tau) x|)$ for $t, \tau \in J, x \in \mathscr{D}(A(0))$. 
(iv) $\quad\left\|(I-\alpha(A(t)-\omega I))^{-1} x-(I-\alpha(A(\tau)-\omega I))^{-1} x\right\| \leqq \alpha|t-\tau| L(\|x\|+|A(\tau) x|)$ for $t, \tau \in J, 0<\alpha<\alpha_{0}, x \in \mathscr{D}(A(0))$.

Here $L: \boldsymbol{R}^{*} \rightarrow \boldsymbol{R}^{*}$ is an increasing function. Then

$$
U(t, s) x=\lim _{n \rightarrow \infty} \prod_{i=1}^{n}\left[I+(t-s) n^{-1}\left(A\left(s+i(t-s) n^{-1}\right)-\omega I\right)\right]^{-1} x
$$

exists for $x \in C$ and $0 \leqq s \leqq t \leqq T$. Here the product is an ordered product: $\prod_{i: 1}^{n} T_{i} x=T_{n} T_{n-1} \cdots T_{1} x$. For $x \in C$ and $0 \leqq r \leqq s \leqq t \leqq T$ we have

(a) $U(t, s): C \rightarrow C$,

(b) $U(t, s) U(s, r) x=U(t, r) x, U(t, t) x=x$,

(c) $\|U(t, s)\|_{\text {Lip }} \leqq e^{\omega(t-s)}$,

(d) $\lim _{t \rightarrow s} U(t, r) x=U(s, r) x$.

$U=\{U(t, x): 0 \leqq s \leqq t \leqq T\}$ is called the evolution operator determined by $A(\cdot) . \quad u$ defined by $u(t)=U(t, 0) x$ is the candidate for the unique solution of (1.2), although (1.2) need not have a solution in general, even if $A(t)$ does not depend on $t$. (For counterexamples see [4, Section 4].)

THEOREM 5.2. For each $n \in Z^{+}$and each $t \in J=[0, T]$, let $A_{n}(t) \subset X \times X$ be such that all the hypotheses of Theorem 5.1 hold with $\omega, L$, and $\alpha_{0}$ not depending on $n$. Let $C_{n}=\operatorname{cl}\left(\mathscr{D}\left(A_{n}(t)\right)\right)$, assume $\lim _{n \rightarrow \infty} C_{n} \supset C_{0}$, and suppose that for $x_{n} \in C_{n}, \lim _{n \rightarrow \infty} x_{n}=x_{0} \in C_{0}$ we have

$$
\lim _{n \rightarrow \infty}\left(I-\alpha\left(A_{n}(t)-\omega I\right)\right)^{-1} x_{n}=\left(I-\alpha\left(A_{0}(t)-\omega I\right)\right)^{-1} x_{0}
$$

for $t \in J, 0<\alpha<\alpha_{0}$. Finally assume that if $x_{n} \in \mathscr{D}\left(A_{n}(0)\right), \lim _{n \rightarrow \infty} x_{n} \in C_{0}$, and $t \in J$, then there is a $y_{n} \in A_{n}(0) x_{n}$ such that $\lim _{n \rightarrow \infty} y_{n}$ exists if and only if there is a $z_{n} \in A_{n}(t) x_{n}$ such that $\lim _{n \rightarrow \infty} z_{n}$ exists. Let $U_{n}=\left\{U_{n}(t, s): 0 \leqq s \leqq t \leqq T\right\}$ be the evolution operator determined by $A_{n}(\cdot)$. Then

$$
\lim _{n \rightarrow \infty} U_{n}(t, s) x_{n}=U_{0}(t, s) x_{0}
$$

whenever $x_{n} \in C_{n}, \lim _{n \rightarrow \infty} x_{n}=x_{0}$; moreover, the limit is uniform in $t$ on $[s, T]$.

We shall use the notation of the proof of Theorem 3.2. For $t \in J$ define $\mathcal{A}(t) \subset \mathscr{X} \times \mathscr{X}$ by $:(\underline{x}, \underline{y}) \in \mathcal{A}(t)$ (where $\underline{x}, \underline{y} \in \mathscr{X}$ ) if and only if $x_{n} \in \mathscr{D}\left(A_{n}(t)\right.$ ), $y_{n} \in A_{n}(t) x_{n}$ for all $n \in N$, and $\lim x_{n} \in C_{0}$. Let $\mathscr{B}(t)=\mathcal{A}(t)-\omega I$; then it follows from the proof of Theorem 3.2 that $\mathscr{B}(t)$ is dissipative and $\mathscr{R}(I-\alpha \mathscr{B}(t)) \supset$ $\mathcal{C}=\operatorname{cl}(\mathscr{D}(\mathscr{B}(0)))$ for all sufficiently small $\alpha>0$. Moreover, $\mathscr{D}(\mathscr{B}(t))$ is independent of $t$ by the last hypothesis of the theorem.

Next, let $N(B)=\inf \{\|\underline{x}\|: \underline{x} \in B\}$ for $B \subset \mathcal{H}$. For $t, \tau \in J, \underline{x} \in \mathscr{D}(\mathcal{A}(0))$ we have 


$$
\begin{aligned}
N(\mathcal{A}(t) \underline{x}) & =\sup _{n}\left|A_{n}(t) x_{n}\right| \\
& \leqq \sup _{n}\left(\left|A_{n}(\tau) x_{n}\right|+|t-\tau| L\left(\left\|x_{n}\right\|\right)\left(1+\left|A_{n}(\tau) x_{n}\right|\right)\right) \quad \text { by (iii) } \\
& \leqq N(\mathcal{A}(\tau) \underline{x})+|t-\tau| L(\|\underline{x}\|)(1+N(\mathcal{A}(\tau) \underline{x})) .
\end{aligned}
$$

Next, for $t, \tau \in J, 0<\alpha<\alpha_{0}$, and $\underline{x} \in \mathscr{D}(\mathcal{A}(0))$ we have

$$
\begin{aligned}
\|\|(I-\alpha \mathscr{B}(t))^{-1} \underline{x}-(I-\alpha \mathscr{B}(\tau))^{-1} \underline{x} \| & \\
& =\sup _{n}\left\|\left(I-\alpha B_{n}(t)\right)^{-1} x_{n}-\left(I-\alpha B_{n}(\tau)\right)^{-1} x_{n}\right\| \\
& \leqq \sup _{n}\left(\alpha|t-\tau| L\left(\left\|x_{n}\right\|+\left|A_{n}(\tau) x_{n}\right|\right)\right) \quad \text { by (iv) } \\
& \leqq \alpha|t-\tau|+L(\|\underline{x}\| \mid+N(\mathcal{A}(\tau) \underline{x})) .
\end{aligned}
$$

It follows from Theorem 5.1 that $U=\{U(t, s): 0 \leqq s \leqq t \leqq T\}$, the evolution operator determined by $\mathcal{A}(\cdot)$, exists and satisfies (a)-(d).

We have $\mathcal{U}(t, s) \underline{x}=\left\{U_{n}(t, s) x_{n}\right\}_{1}^{\infty}$ for $\underline{x} \in \mathcal{C}$, and thus (5.2) holds for $\underline{x} \in \mathcal{C}$ (with $x_{0}=\lim _{n \rightarrow \infty} x_{n}$ ). The rest of the proof is very much like that of Theorem. 3.2 , so it will be omitted.

The next approximation theorem will be one in which the solution of the Cauchy problems exist and converge. We shall require an existence theorem of Martin [12], which is a generalization of an earlier result of Kato [8]. The basic assumption is as follows.

(§) ( $\alpha)$ Let $\{A(t): t \in J=[0, T]\}$ be a family of (single-valued) functions: on $X$ such that $A(t)-\omega(t) I$ is dissipative, where $\omega$ is continuously differentiable. on $J, \mathscr{R}(I-\alpha(A(t)-\omega(t) I))=X$ for all $\alpha>0$, and $D=\mathscr{D}(\mathcal{A}(t))$ does not depend on $t$.

( $\beta$ ) Furthermore, if $\left\{x_{n}\right\}_{1}{ }^{\infty} \subset D, \lim _{n \rightarrow \infty} x_{n}=x_{0}$, sup $\left\|A(t) x_{n}\right\|<\infty$, then $x_{0} \in D$. and $w-\lim _{n \rightarrow \infty} A(t) x_{n}=A(t) x_{0}$.

( $\gamma$ ) There is a constant $K$ such that if $Q$ is a bounded subset of $D$ with $\sup \{\|A(t) x\|: t \in J, x \in Q\}<\infty$, then for every $\varepsilon>0, \beta>0$ there exists $a \delta=$ $\delta(\varepsilon, \beta, Q)>0$ such that

$$
\|x-y-b(A(t) x-A(t) y)\| \leqq(1+K b)\|x-y\|+\varepsilon b
$$

for $0<b \leqq \delta,\|x-y\|>\beta, x, y \in Q, t \in J$.

THEOREM 5.3 [12]. Let (\$) hold. Suppose further there is a continuous function $d: J \times J \times \boldsymbol{R}^{+} \rightarrow \boldsymbol{R}^{+}$such that $d(t, s, \cdot)$ is nondecreasing for each $t, s \in J$ and such that

$$
\|A(t) x-A(s) x\| \leqq|t-s| d(t, s,\|x\|) \quad(1+\|A(t) x\|)
$$

for all $t, s \in J, x \in D$. Then there exists an evolution operator $U$ satisfying (a), 
(b), (d) of Theorem 5.1 and

$$
\text { (c') }\|U(t, s)\|_{\text {Lip }} \leqq \exp \left(\int_{s}^{t} \omega(\tau) d \tau\right) \quad \text { for } 0 \leqq s \leqq t \leqq T .
$$

If $s \in J, x \in D$, then $u(\cdot)=U(\cdot, s) x$ is the unique weakly continuously differentiable solution of

$$
u_{w}{ }^{\prime}(t)=A(t) u(t) \quad(s \leqq t \leqq T), u(s)=x
$$

[here $u_{w}{ }^{\prime}$ denotes the weak derivative of $u$ ]; furthermore, $A(\cdot) u(\cdot)$ is Bochner integrable,

$$
u(t)=x+\int_{s}^{t} A(\tau) u(\tau) d \tau
$$

for all $t \in[s, T]$, and the strong derivative $u^{\prime}(t)$ exist for almost all $t$ and $u^{\prime}(t)=A(t) u(t)$ a.e. in $[s, T]$.

This theorem was proved by Kato [8] assuming that $X^{*}$ is uniformly convex, but not assuming $(\beta),(\gamma)$. The above version, due to Martin [12], is a generalization of Kato's result since a dissipative operator $A$ satisfying $\mathscr{R}(I-\alpha A)=X$ for all $\alpha>0$ automatically satisfies $(\beta),(\gamma)$ if $X^{*}$ is uniformly convex (cf. [8, p. 512], [12, p. 416]). However, as Kato has noted, a linear dissipative operator $A$ satisfying $\mathscr{R}(I-\alpha A)=X$ for all $\alpha>0$ need not satisfy $(\beta),(\gamma)$ if $X$ is an arbitrary Banach space.

THEOREM 5.4. For each $n \in Z^{+}$let $\left\{A_{n}(t): t \in J\right\}$ be a family of operators satisfying the hypothesis of Theorem 5.3 with $\omega, K, \delta, d$ being independent of n. Denote $\mathscr{D}\left(A_{n}(t)\right)$ by $D_{n}$. Assume $\alpha>0$,

(i) $\lim _{n \rightarrow \infty}\left(I-\alpha\left(A_{n}(t)-\omega(t) I\right)\right)^{-1} x_{0}=\left(I-\alpha\left(A_{0}(t)-\omega(t) I\right)\right)^{-1} x_{0}$ for all $x_{0} \in X, t \in J$,

(ii) if for $n \in N, t \in J, x_{n} \in D_{n}, \lim _{n \rightarrow \infty} x_{n}=x_{0}$ exists and $\sup _{n}\left\|A_{n}(t) x_{n}\right\|<\infty$, then $x_{0} \in D_{0}$ and $\lim _{n \rightarrow \infty} A_{n}(t) x_{n}=A_{0}(t) x_{0}$. Then $\lim _{n \rightarrow \infty} U_{n}(t, s) x_{n}=U_{0}(t, s) x_{0}$, uniformly for $t \in[s, T]$, where $U_{n}$ is the evolution operator determined by $A_{n}(\cdot)$, whenever $\left\{x_{n}\right\}_{1}{ }^{\infty}$ is a sequence in $X$ converging to $x_{0}$ and satisfying: for each $\varepsilon>0$ there is a convergent sequence $\left\{x_{n}{ }^{\varepsilon}\right\}_{1}^{\infty}$ such that $\left\|x_{n}-x_{n}{ }^{\varepsilon}\right\|<\varepsilon, x_{n} \in D_{n}$ for all $n \in N$, and $\sup _{n}\left\|A_{n}(0) x_{n}{ }^{\varepsilon}\right\|<\infty$.

The unsatisfactory feature of the above theorem is condition (ii) which is rather restrictive. Theorem 5.4, like all of our other approximation theorems, is deduced from an existence theorem (Theorem 5.3 in this case) by viewing a convergent sequence of evolution operators on $X$ as a single evolution operator on $\mathcal{X}$. We omit the details of proof except for the observation that the dual space of $\mathscr{X}$ is

$$
\mathscr{X}^{*}=\left\{\underline{\phi}:\left\{\phi_{n}\right\}_{0}^{\infty} \subset X^{*}:\|\underline{\phi}\|=\sum_{n-0}^{\infty}\left\|\phi_{n}\right\|<\infty\right\},
$$

and for $\underline{\phi} \in \mathfrak{X}^{*}, \underline{x} \in \mathfrak{X}$, 


$$
\underline{\phi}(\underline{x})=\sum_{n=1}^{\infty} \phi_{n}\left(x_{n}\right)+\phi_{0}\left(\lim _{n \rightarrow \infty} x_{n}\right) .
$$

This follows from a representation theorem of Singer [19], [20].

We thank Robert H. Martin, Jr., for showing us how to fix up an error in an early version of the proof of Theorem 5.4.

Finally, our method of proof in the linear case yields the following result.

THEOREM 5.5. For each $n \in Z^{+}$let $\left\{A_{n}(t): t \in J\right\}$ be a family of linear operators on $X$ satisfying $(\alpha)$ of $(\S)$, where $\omega$ does not depend on $n$. Suppose $B_{n}=A_{n}(\cdot)\left(I-\left(A_{n}(0)-\omega_{0} I\right)\right)^{-1}$ is strongly continuously differentiable on $J$, where $\omega_{0}=\sup \{|\omega(t)|: t \in J\}$; and the strong derivative of $B_{n}$ is bounded independent of $t \in J, n \in Z^{+}$. Finally assume that

$$
\lim _{n \rightarrow \infty}\left(I-\alpha\left(A_{n}(t)-\omega(t) I\right)\right)^{-1} x=\left(I-\alpha\left(A_{0}(t)-\omega(t) I\right)\right)^{-1} x
$$

for all $x \in X, t \in J$. Let $U_{n}$ denote the evolution operator determined by $A_{n}$. Then

$$
\lim _{n \rightarrow \infty} U_{n}(t, s) x_{n}=U_{0}(t, x) x_{0},
$$

uniformly for $t \in[s, T]$, whenever $\left\{x_{n}\right\}_{1}^{\infty}$ is a convergent sequence in $X$ with limit $x_{0}$. Let $\left\{f_{1}\right\}_{0}^{\infty}$ be a boundedly convergent sequence of continuous $X$-valued functions on $J$, and let $\lim _{n \rightarrow \infty} x_{n}=x_{0}$. Then

$$
\lim _{n \rightarrow \infty} u_{n}(t)=u_{0}(t)
$$

for $t \in[s, T]$, where $u_{n}$ is the unique mild solution of

$$
u_{n}{ }^{\prime}(t)=A_{n}(t) u_{n}(t)+f_{n}(t) \quad(s \leqq t \leqq T), u_{n}(s)=x_{n} ;
$$

and the convergence is uniform if the convergence of $\left\{f_{n}\right\}_{1}{ }^{\infty}$ is uniform.

"Mild solution" means solution of the associated integral equation. This theorem complements some approximation theorems of Kato [9].

REMARKS. (1) After this paper was submitted we learned of some related preprints. Brezis and Pazy [25] overlaps with Section 3. Fitzgibbon [27], [28] and Crandall and Pazy [26] overlap with Section 5. Our proofs are quite different from those of the above authors. We thank these authors for sending us their papers, and we thank Crandall and Pazy for some useful comments concerning the present paper.

(2) If $T_{n}(t)=\lim _{m \rightarrow \infty}\left(I-t m^{-1} A_{n}\right)^{-1}$ and if (3.1) holds, then we cannot expect (3.2) to hold since $T_{n}$ does not determine $A_{n}$ uniquely (cf. [4]). Thus the sufficient condition (3.2) cannot be necessary in general. However, if the dual space $X^{*}$ is uniformly convex, then $T_{n}$ determines $A_{n}$ uniquely (cf. Brezis [24]), and the necessity of (3.2) might be valid in this case. Bénilan 
[23] established the necessity of (3.2) in the Hilbert space case.

(3) Finally, in the case when both $X$ and $X^{*}$ are uniformly convex, Martin [29] has characterized the generators $G$ of semigroups $T \in Q_{\omega}(C)$ (where $C$ is a closed subset of $X$ ) having the property that $c l(\mathscr{D}(G))=C$.

\author{
Department of Mathematics \\ Tulane University \\ New Orleans, Louisiana 70118
}

\title{
References
}

[1] H. Brezis, M. G. Crandall and A. Pazy, Perturbations of nonlinear maximal monotone sets in Banach space, Comm. Pure Appl. Math., 23 (1970), 123-1.44.

[2] H. Brezis and A. Pazy, Semigroups of nonlinear contractions on convex sets, J. Functional Analysis, 6 (1970), 237-281.

[3] P. R. Chernoff, Semigroup Product Formulas and Addition of Unbounded Opera. tors, Ph. D. Thesis, Harvard Univ., 1968.

[4] M. G. Crandall and T.M. Liggett, Generation of semi-groups of nonlinear transformations on general Banach spaces, Amer. J. Math., 93 (1971), 265-298.

[5] M. G. Crandall and A. Pazy, Semi-groups of nonlinear contractions and dissipative sets, J. Functional Analysis, 3 (1969), 376-418.

[6] J. A. Goldstein, Semigroups of Operators and Abstract Cauchy Problems, Tulane University Lecture Notes, 1970.

[7] J. A. Goldstein, Some counterexamples involving self-adjoint operators, Rocky Mtn. Math. J. (to appear).

[8] T. Kato, Nonlinear semigroups and evolution equations, J. Math. Soc. Japan, 19 (1967), 508-520.

[9] T. Kato, Approximation theorems for evolution equations, Lectures in Differential Equations, Volume II, Van Nostrand Reinhold, New York (1969), 115-124.

[10] T. Kato, Accretive operators and nonlinear evolution equations in Banach spaces, Proc. Symp. Pure Math., 18, Part I, Amer. Math. Soc., Providence, R. I., 1970, 138-161.

[11] J. Kisyński, A proof of the Trotter-Kato theorem on approximation of semigroups, Coll. Math., 18 (1967), 181-184.

[12] R.H. Martin, Jr., The logarithmic derivative and equations of evolution in a Banach space, J. Math. Soc. Japan, 22 (1970), 411-429.

[13] R. H. Martin, Jr., A global existence theorem for autonomous differential equations in a Banach space, Proc. Amer. Math. Soc., 26 (1970), 307-314.

[14] J. Mermin, An exponential limit formula for nonlinear semigroups, Trans. Amer. Math. Soc., 150 (1970), 469-476.

[15] I. Miyadera, On the convergence of nonlinear semi-groups, Tôhoku Math. J., 21 (1969), 221-236.

[16] I. Miyadera, On the convergence of nonlinear semi-groups II, J. Math. Soc. Japan, 21 (1969), 403-412.

[17] I. Miyadera and S. Ôharu, Approximation of semi-groups of nonlinear operators, to appear.

[18] A. Pazy, Semi-groups of nonlinear contractions in Hilbert space, Problems in 
Non-linear Analysis, Edizione Cremonese, Rome, 1971, 343-430.

[19] I. Singer, Linear functionals on the space of continuous mappings of a compact space into a Banach space (in Russian), Revue Math. Pures et Appl., 2 (1957), 301-315.

[20] I. Singer, Les duals des certain espaces de Banach de champs de vecteurs, I, II, Bull. Sci. Math., 82 (1958), 29-40; 83 (1959), 73-96.

[21] R. A. Struble, Nonlinear Differential Equations, McGraw-Hill, New York, 1962.

[22] G. F. Webb, Nonlinear evolution equations and product integration in Banach spaces, Trans. Amer. Math. Soc., 148 (1970), 273-282.

[23] P. Bénilan, Une remarque sur la convergence des semi-groupes non linéaires, C. R. Acad. Sc. Paris, 272 (1971), 1182-1184.

[24] H. Brezis, On a problem of T. Kato, Comm. Pure Appl. Math., 24 (1971), 1-6.

[25] $\mathrm{H}$. Brezis and A. Pazy, Convergence and approximation of semigroups of non. linear operators in Banach spaces, J. Functional Analysis, to appear.

[26] M. G. Crandall and A. Pazy, Nonlinear evolution equations in Banach spaces, to appear.

[27] W. E. Fitzgibbon, Approximation of nonlinear evolution equations, to appear.

[28] W.E. Fitzgibbon, Time dependent nonlinear Cauchy problems in Banach spaces, to appear.

[29] R.H. Martin, Jr., Generating an evolution system in a class of uniformly convex Banach spaces, to appear.

[30] I. Miyadera, Some remarks on semi-groups of nonlinear operators, Tôhoku Math. J., 23 (1971), 245-258. 